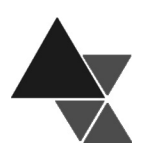

\title{
Segurança Alimentar e Nutricional na Região Centro-Oeste: particularidades e contrastes
}

\author{
Sarah Guerra Gama Tinoco, Juliana Frossard Ribeiro Mendes ${ }^{2}$, Aline Cristino Figueiredo ${ }^{2}$, \\ Ana Paula Rezende Costa ${ }^{3}$, Marília Mendonça Leão ${ }^{4}$, Leonor Maria Pacheco Santos ${ }^{5}$
}

Trata-se de um estudo descritivo baseado em dados secundários que analisou a situação de Segurança Alimentar e Nutricional (SAN) no Centro-Oeste (CO), a partir de indicadores da Matriz de monitoramento proposta pelo Conselho Nacional de Segurança Alimentar e Nutricional. Verificou-se que a região apresenta a maior produção de soja e carne bovina do país e a maior porcentagem de gastos com alimentação total. Em contraste, possui a maior concentração de renda, a menor disponibilidade de calorias do país e baixa aquisição de frutas e hortaliças. Para algumas culturas de frutas e vegetais registrou-se maior percentual de amostras contaminadas por agrotóxicos na Região CO, comparado à situação nacional. Para ambos os sexos a prevalência de déficit de peso em adultos foi maior se comparada à média do país; para o sexo masculino constatou-se maior prevalência de excesso de peso e obesidade e para as mulheres, uma menor prevalência, em comparação aos dados nacionais. Indicadores de baixo peso ao nascer, mortalidade infantil, aleitamento materno e acesso ao pré-natal foram melhores do que os do Brasil. Apesar do CO ocupar posição de liderança na produção agropecuária, é uma região de contrastes marcantes e muitos indicadores apontaram para situações que podem acarretar insegurança alimentar e nutricional.

Palavras-chave: Segurança Alimentar e Nutricional, Centro-Oeste brasileiro, monitoramento.

\section{Food and Nutrition Security in Brazil's Central-West Region: Peculiarities and contrasts}

This study evaluated the food and nutritional security in Brazil's Central-West (CW) region based on indicators which reflect its different dimensions. It was a descriptive study, employing secondary data based on the monitoring Matrix proposed by the Brazilian National Food and Nutritional Security Council. Results showed that the CW region presented the largest soy and beef production of the country and the largest percent on food of total household expenditures. But in contrast the CW region presented the worst income distribution, the lowest caloric availability and the lowest acquisition of fruit and vegetables in the country. For some produce, the largest number of fruits and vegetables contaminated by pesticides was found in the $\mathrm{CW}$ region compared to national figures. For both sexes the prevalence of underweight was higher in adults compared to the national average, for males there was greater prevalence of overweight and obesity and a lower prevalence for women, compared to national data. Birth weight, infant mortality, breast feeding and pre-natal accessibility indicators were better than the national figures. Although its leadership in agribusiness, the CW region presents marked contrasts and many indicators showed that the population is exposed to food and nutrition insecurity.

Key-words: food and nutrition security, Brazil's Center West, monitoring.

\footnotetext{
${ }^{1}$ Mestre em Nutrição Humana pela Universidade de Brasília (UNB), Programa de Pós-Graduação em Nutrição Humana. Correspondência: Secretaria de Estado de Saúde do Distrito Federal. SGAN 601 Norte, Blocos O/P, Sala 21, Gerência de Doenças e Agravos não Transmissíveis - Asa Norte - Brasília, Distrito Federal (DF). CEP: 70.830-010. Tel: (61) 3323 3056, Fax: (61) 3323 3056.E-mail: sarahggt@hotmail.com.

${ }^{2}$ Mestre em Nutrição Humana pela UNB, Programa de Pós-Graduação em Nutrição Humana.

${ }^{3}$ Mestre em Ciências Médicas pela UNB, Programa de Pós-Graduação em Ciências Médica.

${ }^{4}$ Mestre em Nutrição Humana pela UNB, Ação Brasileira pela Nutrição e Direitos Humanos.

${ }^{5}$ Pós-doutora e Professora adjunta da UNB, Programa de Pós-Graduação em Nutrição Humana. Departamento de Saúde Coletiva.
} 


\section{INTRODUÇÃO}

O tema Segurança Alimentar e Nutricional (SAN) vem ganhando relevância nas últimas décadas, sendo alvo de reflexão e discussão em reuniões de cúpula e no meio acadêmico [1]. Na América Latina há uma tradição de estudos sobre a problemática da fome e Insegurança Alimentar (IA). Desde a década de 30, esta questão já era analisada de forma inquietante por pensadores como Josué de Castro, um dos precursores do tema no Brasil. Nos anos 80, a utilização do termo pouco havia evoluído e limitava-se a observar o estado nutricional dos indivíduos, sem que fossem levados em consideração outros determinantes e indicadores relacionados à autonomia alimentar dos mesmos [2].

Os debates sobre a questão, a partir dos anos de 90, assumem outra dimensão e ganham novos objetivos. No início da década, o direito à alimentação passou a ser reconhecido e foi estabelecido na Carta dos Direitos Humanos, da qual o Brasil é signatário. Essa mudança, fundamental na forma de encarar o Direito Humano à Alimentação Adequada (DHAA), colocou o Estado na posição de provedor e responsável pelo bem-estar alimentar de sua população [3]. Em 1996, os países membros das Nações Unidas, entre os quais o Brasil, se reuniram em Roma, na Cúpula Mundial da Alimentação e, a partir de um esforço conjunto, construíram uma nova definição de segurança alimentar [4].

Mais recentemente, a II Conferência Nacional de Segurança Alimentar e Nutricional (CNSAN), realizada em 2004, no Brasil, estabeleceu que SAN é:

\begin{abstract}
A realização do direito de todos ao acesso regular e permanente a alimentos de qualidade, em quantidade suficiente, sem comprometer o acesso a outras necessidades essenciais, tendo como base práticas alimentares promotoras da saúde, que respeitem a diversidade cultural e que sejam social, econômica e ambientalmente sustentáveis (2004, p. 4) [5].
\end{abstract}

Mais adiante, a Declaração Final da III CNSAN, ocorrida em 2007, reafirma que o objetivo da SAN implica em uma concepção de desenvolvimento socioeconômico que questiona os componentes do modelo hegemônico geradores de desigualdade, pobreza e fome e com impactos negativos sobre o meio ambiente e a saúde [6].
Esta concepção ampla envolve um conjunto de questões referentes ao comércio de alimentos, à soberania alimentar, à conformação da pobreza e da desigualdade em cada sociedade, à qualidade sanitária e nutricional dos alimentos, à privatização dos recursos ambientais e da base genética do sistema agroalimentar, à degradação ambiental, ao processo saúde-doença e ao perfil de consumo alimentar de risco à saúde. Sendo assim alguns autores consideram que a SAN só poderia ser definida a partir de uma ótica relativa e não absoluta [7].

A identificação da situação de SAN deve abranger análises pautadas por múltiplas dimensões. Para tanto, é necessária uma abordagem que incida sobre os diversos determinantes da SAN, sendo essencial articular a dimensão alimentar (da produção, comercialização e consumo) e a dimensão nutricional (da utilização biológica do alimento pelo organismo e sua relação com a saúde). Não há como promover um consumo adequado sem incidir sobre o sistema produtivo que determina a forma como o alimento é produzido, comercializado e disponibilizado e sobre os fatores que condicionam o consumo, como custo, valores, meios de comunicação, dentre outros [7].

Atualmente, o Brasil tem uma produção anual de 120 milhões de toneladas de grãos sendo autosuficiente na maior parte dos alimentos básicos consumidos. O país apresenta, ainda, uma disponibilidade de 3.000 calorias e de 100 gramas diárias de proteínas por habitante, mais da metade oriundas de alimentos de origem animal. No âmbito do mercado interno, dispõe-se de um excedente de $40 \%$ de oferta calórica e de $50 \%$ no que se refere às necessidades efetivas de proteínas. Deste ponto de vista, poucos países têm uma situação tão confortável $[8]$.

A Região Centro-Oeste (CO), por sua vez, destaca-se por possuir a fronteira agropecuária que mais cresce no mundo, com indicadores que rivalizam com o milagre econômico da Índia e da China, atingindo índices anuais de incremento de $7 \%$ a $10 \%$ [8]. Paradoxalmente alguns estudos indicaram que uma proporção importante da população desta região encontra-se em situação de IA [0,10].

Considerando o número ainda limitado de trabalhos científicos disponíveis sobre a Região CO [11], verificou-se a necessidade da realização deste estudo para melhor caracterização e conhecimento dos processos que envolvem o tema na região. $\mathrm{O}$ objetivo 
do presente trabalho foi, portanto, analisar a situação de SAN da Região CO, a partir de indicadores de suas diferentes dimensões conceituais.

\section{METODOLOGIA}

Trata-se de um estudo descritivo baseado em dados secundários, que utilizou como arcabouço conceitual a Matriz proposta pelo grupo técnico executivo de indicadores e monitoramento do Conselho Nacional de Segurança Alimentar e Nutricional (CONSEA) com o objetivo de monitorar o cenário da SAN no Brasil e a realização progressiva do DHAA [6,12]. Essa Matriz abrange sete dimensões de SAN: (1) produção de alimentos; (2) disponibilidade de alimentos; (3) renda, acesso e gasto com alimentos; (4) acesso à alimentação adequada; (5) saúde e acesso a serviços de saúde; (6) educação; (7) políticas públicas que promovem a SAN. Não foi possível analisar os indicadores da dimensão 2 pela dificuldade de acesso a dados desagregados por região. Assim sendo optou-se por avaliar, na Região $\mathrm{CO}$, os indicadores de seis dimensões, comparando os resultados entre os Estados da região, com dados nacionais e, em alguns casos, com outras regiões do Brasil. Todos os indicadores utilizados, para cada dimensão analisada, foram propostos pela Matriz de SAN do CONSEA.

Para a análise das seis dimensões em estudo os dados secundários foram obtidos a partir das fontes indicadas no Quadro [13-22]. Na dimensão "produção de alimentos" foram avaliados os principais produtos agropecuários do $\mathrm{CO}$ e do Brasil previstos na Matriz do CONSEA. No caso da dimensão "acesso à alimentação adequada" para obter dados dos dois primeiros indicadores foi preciso recorrer à publicação "Análise da disponibilidade de alimentos e estado nutricional POF 2002-2003" [17], tendo em vista que ainda não foram disponibilizadas informações dessa natureza coletadas pela última POF (2008/2009). Os dados de IA foram obtidos por meio das Pesquisas Nacionais por Amostra de Domicílios (PNAD - 2004 e 2009) [9], que empregaram a Escala Brasileira de Insegurança Alimentar (EBIA).

$\mathrm{Na}$ dimensão "saúde e acesso a serviços de saúde" foram avaliados os índices antropométricos para diferentes etapas do curso da vida, além de outros indicadores propostos na Matriz de SAN. Para crianças menores de 5 anos foi avaliada a prevalência de déficit de Peso para Idade (P/I) segundo dados da Pesquisa
Nacional de Demografia e Saúde da Criança e da Mulher (PNDS) de 2006 [18], a qual também forneceu informações sobre o déficit de Altura para Idade (A/I). Avaliou-se o estado nutricional dos adolescentes por meio da prevalência de déficit de peso, excesso de peso e obesidade, a partir do Índice de Massa Corporal (IMC) para idade, calculadas com base na distribuição da referência da Organização Mundial de Saúde (OMS) e para os adultos foi estudado o IMC e analisada a proporção de déficit de peso, excesso de peso e obesidade, ambos obtidos na POF 2008-2009 [19] (Quadro).

Para analisar o acesso a alimentos seguros dentro do contexto da SAN, foram utilizados os dados de alimentos contaminados por agrotóxicos gerados pelo Programa de Análise de Resíduos de Agrotóxicos em Alimentos (PARA) de 2008, da Agência Nacional de Vigilância Sanitária (ANVISA) (Quadro).

\section{RESULTADOS E DISCUSSÃO}

\section{Dimensão 1: Produção de alimentos}

O país é grande produtor de alimentos, sendo que o cultivo dos grãos está distribuído de forma desigual entre as diferentes regiões, principalmente no que se refere ao arroz, à soja e ao trigo. A soja tem concentração de produção importante nas Regiões CO e Sul. No CO, destaca-se que somente a produção de soja do Estado do Mato Grosso é quase igual a toda a produção da Região Sul, a segunda maior produtora do grão no Brasil [13].

Com relação à produção pecuária, a Região CO destaca-se principalmente pela elevada participação na produção de carne bovina em âmbito nacional, sendo responsável por mais de um terço $(33,7 \%)$ dos bovinos abatidos no país [14].

$\mathrm{Na}$ Tabela 1, observa-se a produção da pecuária e das principais culturas agrícolas na Região CO e no Brasil.

No entanto, se por um lado a produção de soja e a pecuária conferiram à Região CO posição de destaque no agronegócio, por outro, foram responsáveis pela ocupação de grandes áreas territoriais, prejudicando a agricultura de subsistência e colaborando para o desmatamento e degradação ambiental observados na região. 


\begin{tabular}{|c|c|c|c|}
\hline \multicolumn{4}{|c|}{ Dimensão 1: Produção de alimentos } \\
\hline Indicador & Data base & Fonte & Referência \\
\hline Produção de arroz, feijão, soja e trigo & $2008 / 2009^{a}$ & CONAB & 13 \\
\hline Produção suína, de frango, bovina e de leite & 2009 & IBGE & 14 \\
\hline \multicolumn{4}{|c|}{ Dimensão 3: Renda, acesso e gastos com alimentos } \\
\hline Indicador & Data base & Fonte & Referência \\
\hline $\begin{array}{l}\text { Porcentagem de gastos das famílias com alimentação total, } \\
\text { dentro e fora do domicílio }\end{array}$ & $2008 / 2009$ & IBGE - POF & 15 \\
\hline $\begin{array}{l}\text { Índice de Gini de distribuição do rendimento mensal em } \\
\text { Domićlíios Particulares Permanentes (DPP) com rendimento }\end{array}$ & 2007 & IBGE - PNAD & 16 \\
\hline Proporção por classe de renda domiciliar per capita & 2007 & IBGE - PNAD & 16 \\
\hline \multicolumn{4}{|c|}{ Dimensão 4: Acesso à alimentação adequada } \\
\hline Indicador & Data base & Fonte & Referência \\
\hline $\begin{array}{l}\begin{array}{l}\text { Percentual de macronutrientes no total de calorias na } \\
\text { alimentação domiciliar }\end{array} \\
\end{array}$ & $2002 / 2003$ & IBGE - POF & 17 \\
\hline $\begin{array}{l}\text { Quantidades físicas adquiridas de alimentos para consumo no } \\
\text { domicílio per capita (em kg/ano) }\end{array}$ & $2002 / 2003$ & IBGE - POF & 17 \\
\hline $\begin{array}{l}\text { Proporção de domicílios com Insegurança Alimentar, por tipo } \\
\text { de Insegurança Alimentar }\end{array}$ & 2009 & IBGE - PNAD & 10 \\
\hline \multicolumn{4}{|c|}{ Dimensão 5: Saúde e acesso a serviços de saúde } \\
\hline Indicador & Data base & Fonte & Referência \\
\hline Estado nutricional de crianças $(0-5$ anos $)$ & 2006 & PNDS & 18 \\
\hline $\begin{array}{l}\text { Estado nutricional de adolescentes }(10-19 \text { anos) e de adultos } \\
\text { (20 anos e mais) }\end{array}$ & $2008 / 2009$ & IBGE - POF & 19 \\
\hline Proporção de baixo peso ao nascer (peso $<2.500 \mathrm{~g}$ ) & 2006 & $\begin{array}{c}\text { SINASC } \\
\text { DATASUS - }\end{array}$ & 20 \\
\hline $\begin{array}{l}\text { Taxa de mortalidade infantil (óbitos de nascidos vivos no } 10 \text { ano } \\
\text { de vida por } 1.000 \text { nascidos vivos) }\end{array}$ & 2006 & $\begin{array}{l}\text { SINASC - } \\
\text { DATASUS }\end{array}$ & 20 \\
\hline Prevalência de crianças $<6$ meses em amamentação exclusiva & 2008 & $\begin{array}{l}\text { Ministério } \\
\text { da Saúde }\end{array}$ & 21 \\
\hline Proporção de acesso ao pré-natal & 2005 & $\begin{array}{c}\text { IDB - } \\
\text { DATASUS }\end{array}$ & 20 \\
\hline Proporção de alimentos contaminados por agrotóxicos & 2008 & PARAc & \\
\hline $\begin{array}{l}\text { Percentual de domićlios atendidos por rede geral de } \\
\text { abastecimento de água; serviço de coleta de lixo; dotados de } \\
\text { esgotamento por rede de esgoto/fossa séptica no total de DPP }\end{array}$ & 2005 & IBGE - PNAD & 22 \\
\hline \multicolumn{4}{|c|}{ Dimensão 6: Educação } \\
\hline Indicador & Data base & Fonte & Referência \\
\hline $\begin{array}{l}\text { Número médio de anos de estudo das pessoas com } 10 \text { anos de } \\
\text { idade ou mais }\end{array}$ & 2005 & IBGE - PNAD & 22 \\
\hline $\begin{array}{l}\text { Proporção de crianças e adolescentes entre } 5 \text { e } 17 \text { anos que não } \\
\text { frequentavam a escola }\end{array}$ & 2005 & IBGE - PNAD & 22 \\
\hline $\begin{array}{l}\text { Taxa de analfabetismo e analfabetismo funcional entre as } \\
\text { pessoas com } 15 \text { anos de idade ou mais }\end{array}$ & 2005 & IBGE - PNAD & 22 \\
\hline \multicolumn{4}{|c|}{ Dimensão 7: Políticas públicas que promovem a SAN } \\
\hline Indicador & Data base & Fonte & Referência \\
\hline Bolsa Família; Cestas; PAAd; Equipamentos & Nov 2009 & MDS & 33 \\
\hline
\end{tabular}

a Até agosto de 2009; b Sistema de Informações de Nascidos Vivos (SINASC); c Dados de serviço da Agência Nacional de Vigilância

Sanitária (ANVISA) - Programa de Análise de Resíduos de Agrotóxicos em Alimentos (PARA); d Programa de Aquisição de Alimentos da Agricultura Familiar (PAA).

Quadro. Dimensões, indicadores e fonte de dados sobre Segurança Alimentar e Nutricional na Região Centro-Oeste e no Brasil. 
Tabela 1. Principais produtos agropecuários na Região Centro-Oeste e no Brasil, 2008/2009.

\begin{tabular}{|c|c|c|c|}
\hline Produto & Centro-Oeste & Brasil & $\begin{array}{l}\text { Proporção em } \\
\text { relação à produção } \\
\text { nacional }(\%)\end{array}$ \\
\hline Arroz $^{a}$ & $1.223,7$ & $12.809,3$ & 9,5 \\
\hline Feijão total ${ }^{a}$ (1a, $2 \underline{a}$ e $3 \underline{a}$ safra) & 456,1 & $3.763,6$ & 12,1 \\
\hline Milho total ${ }^{a}$ (1 a e 2 a safra) & $14.594,6$ & $51.381,2$ & 28,4 \\
\hline Soja $\mathrm{a}^{\mathrm{a}}$ & $29.202,4$ & $57.618,4$ & 50,7 \\
\hline Trigo $^{a}$ & 167,0 & $6.015,6$ & 2,8 \\
\hline Bovinos $^{b}$ & $2.249 .779,0$ & 6. $678.860,0$ & 33,7 \\
\hline Suínosb & $962.397,0$ & $7.376 .143,0$ & 13,0 \\
\hline Frangos ${ }^{b}$ & 153.142.194,0 & 1.245.484.257,0 & 12,3 \\
\hline Leite $^{c}$ & $771.761,0$ & $4.899 .673,0$ & 15,7 \\
\hline
\end{tabular}

a Em mil toneladas; b Número de cabeças abatidas; c Em mil litros de leite cru ou resfriado adquirido. Fontes: Grãos, Companhia Nacional de Abastecimento (CONAB), Safra 2008/2009 (até abril de 2009); Produtos pecuários Estatística da Produção Pecuária, Instituto Brasileiro de Geografia e Estatística (IBGE), março de 2009.

\section{Dimensão 3: Renda, acesso e gastos com alimentos}

O Índice de Gini é a medida do grau de concentração de uma determinada distribuição, cujo valor varia de zero (a perfeita igualdade) até um (a desigualdade máxima). De acordo com esse índice, na Região CO houve uma redução na concentração de renda no período de 2004 a 2006 (de 0,553 para 0,543 ), seguida de um aumento entre 2006 e 2007 (de 0,543 para 0,552). Por outro lado, no Brasil, no mesmo período, observa-se uma redução contínua deste índice, de 0,535 para 0,521. Quando comparado ao índice nacional e das demais regiões, em qualquer ano, o Índice de Gini no CO sempre foi o mais alto, demonstrando uma maior desigualdade da distribuição do rendimento mensal dos domicílios particulares permanentes nessa região, segundo a PNAD 2007 [16].

Em relação ao indicador renda domiciliar per capita, observa-se que mais da metade $(51,5 \%)$ das famílias residentes em domicílios particulares na Região CO possuem renda mensal familiar per capita de até 1 salário mínimo ou não têm rendimento, semelhante aos dados nacionais $(53,2 \%)$. Destaca-se que o percentual de famílias com mais de cinco salários mínimos de rendimento familiar mensal per capita $(7,6 \%)$ é maior do que o encontrado no Brasil (5,5\%), enquanto o percentual de famílias com até $1 / 4$ de salário mínimo de rendimento familiar mensal per capita é menor $(4,4 \%)$ que o encontrado no país (7,9\%), segundo a PNAD 2007 [16]. Constata-se que apesar deste bom resultado, a distribuição de renda na Região CO é a pior do Brasil, de acordo com o Índice de Gini, fato que pode impactar de forma negativa no acesso aos alimentos e, consequentemente, na SAN de parte dessa população.

Em relação à despesa monetária e não monetária familiar com alimentação total, dentro e fora do domicílio, observa-se que a Região CO $(14,07 \%)$ se aproxima da média nacional (16,05\%). Ao comparar o $\mathrm{CO}$ às demais regiões brasileiras, este apresenta a menor porcentagem de gasto das famílias com alimentação total $(14,07 \%)$, sendo a segunda região com maior porcentagem de gasto das famílias com alimentação fora do domicílio $(30,12 \%)$ e a penúltima região com menor porcentagem de gasto das famílias com alimentação no domićlio (69,88\%), segundo a Pesquisa de Orçamentos Familiares (POF) 2008-2009 [15].

\section{Dimensão 4: Acesso à alimentação adequada}

Quanto à quantidade de alimento adquirida per capita por ano para consumo no domicílio (em $\mathrm{kg} / \mathrm{ano}$ ) verificou-se que em 2002-2003 a aquisição de alimentos diferia de forma expressiva nas regiões do país, especialmente para determinados grupos de alimentos como as hortaliças, frutas, pescados, leite e cremes de leite (Figura 1). Na Região CO, a aquisição de cereais e leguminosas ultrapassava a média nacional, enquanto a quantidade de alimentos ricos em vitaminas e sais minerais como hortaliças e frutas foi baixa, sendo a 
segunda menor do Brasil; quanto à aquisição de pescados, a população do $\mathrm{CO}$ apresentou a menor proporção no país. Destaca-se que as quantidades per capita adquiridas de óleos e gorduras na região $(12,54$ $\mathrm{kg} / \mathrm{ano})$ foram superiores a média do país $(10,22$ $\mathrm{kg} / \mathrm{ano}){ }^{[17]}$.

Chamou atenção a participação do arroz na disponibilidade alimentar da Região CO, que excedeu em aproximadamente duas vezes a participação observada nas demais regiões do país. No entanto, o $\mathrm{CO}$ apresentou valores de disponibilidade de hortaliças menores do que as Regiões Sul e Sudeste, sendo que para frutas a disponibilidade foi muito próxima a das Regiões Norte e Nordeste. É interessante observar que, apesar do CO ser o maior produtor de carne do país, a disponibilidade domiciliar desse alimento na região (23,628 kg/ano) está abaixo da média nacional (25,237 $\mathrm{kg} / \mathrm{ano}$ ), sendo a segunda menor do Brasil. Ressalta-se, por fim, que a disponibilidade total de calorias (kcal/dia per capita) da Região CO é a menor do país (1.714 Kcal).

Em relação à composição da dieta relativa aos macronutrientes, verifica-se na Região CO adequação do teor protéico, excesso do teor de gorduras (mais do que $30 \%$ das calorias totais) e excesso do teor de açúcares. Embora haja adequação do teor protéico na alimentação da população residente no CO $(11,9 \%$ das calorias totais), o valor é o menor dentre todas as outras regiões (variação de 12,1\% a 14,1\%) [17].

De acordo com os dados das PNADs 2004 e 2009, que aplicaram a EBIA, verificou-se que a prevalência de qualquer tipo de IA (leve, moderada e grave) na Região CO manteve-se praticamente estável no período, passando de $31,1 \%$, para $30,1 \%$ enquanto que a prevalência no Brasil diminuiu de 34,8\% para 30,2\% (Figura 2) [10]. Segundo análise da PNAD 2009 as diferenças entre as Unidades da Federação de uma mesma grande região foram pequenas no Sul, Sudeste e no $\mathrm{CO}$, onde a maior prevalência de IA grave foi de $5,1 \%$, em Goiás, e a menor, de 2,7\%, no Distrito Federal e no Mato Grosso.

\section{Dimensão 5: Saúde e acesso a serviços de saúde}

\section{Estado nutricional segundo o ciclo de vida}

Analisando-se os dados sobre crianças menores de 5 anos apresentados pela PNDS de 2006/2007 [18] (Tabela 2), a prevalência de déficit de $\mathrm{P} / \mathrm{I}$ e de $\mathrm{A} / \mathrm{I}$ para a Região $\mathrm{CO}$ foi inferior à média do Brasil (número de crianças avaliadas $=863$ no $\mathrm{CO}$ e 4.367 no Brasil). Foi observado que a população do

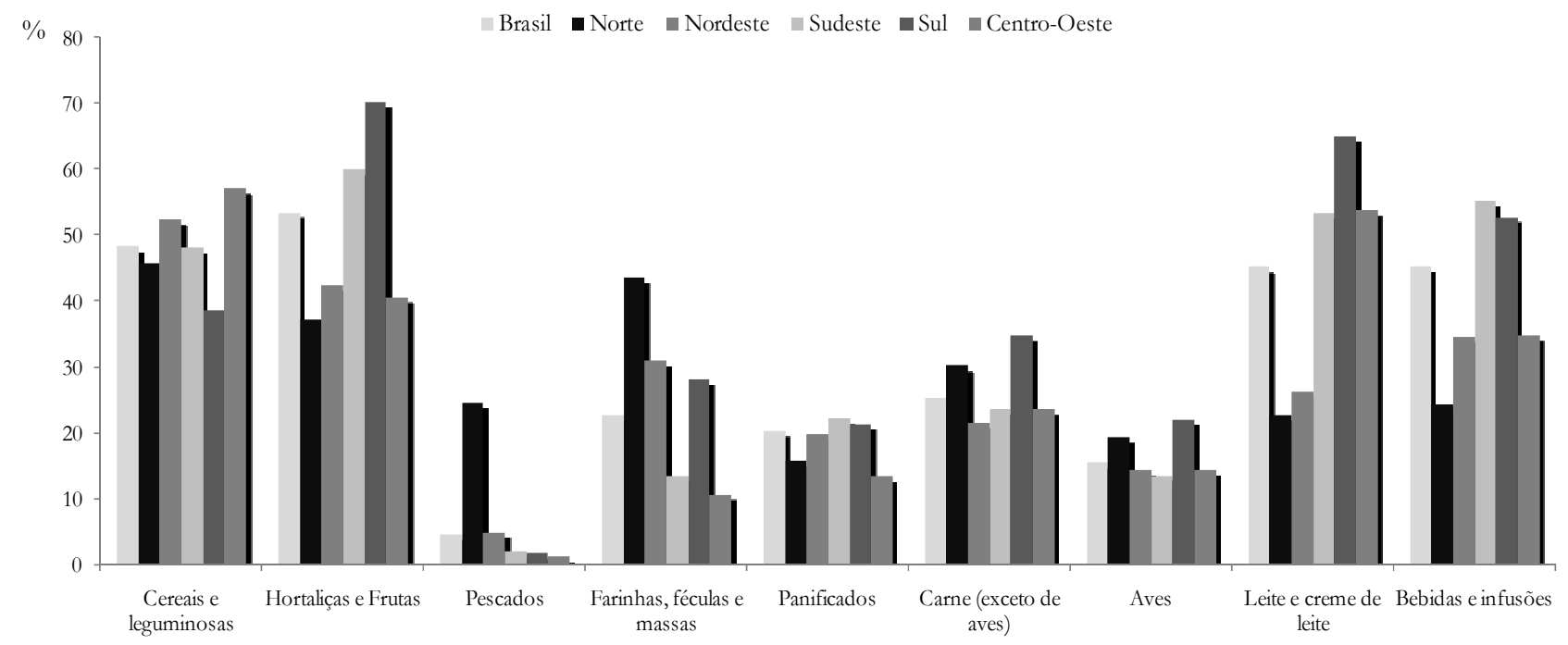

Figura 1. Aquisição alimentar domiciliar per capita anual, por grandes regiões do Brasil, segundo grupo de produtos. Brasil, 2002-2003. 


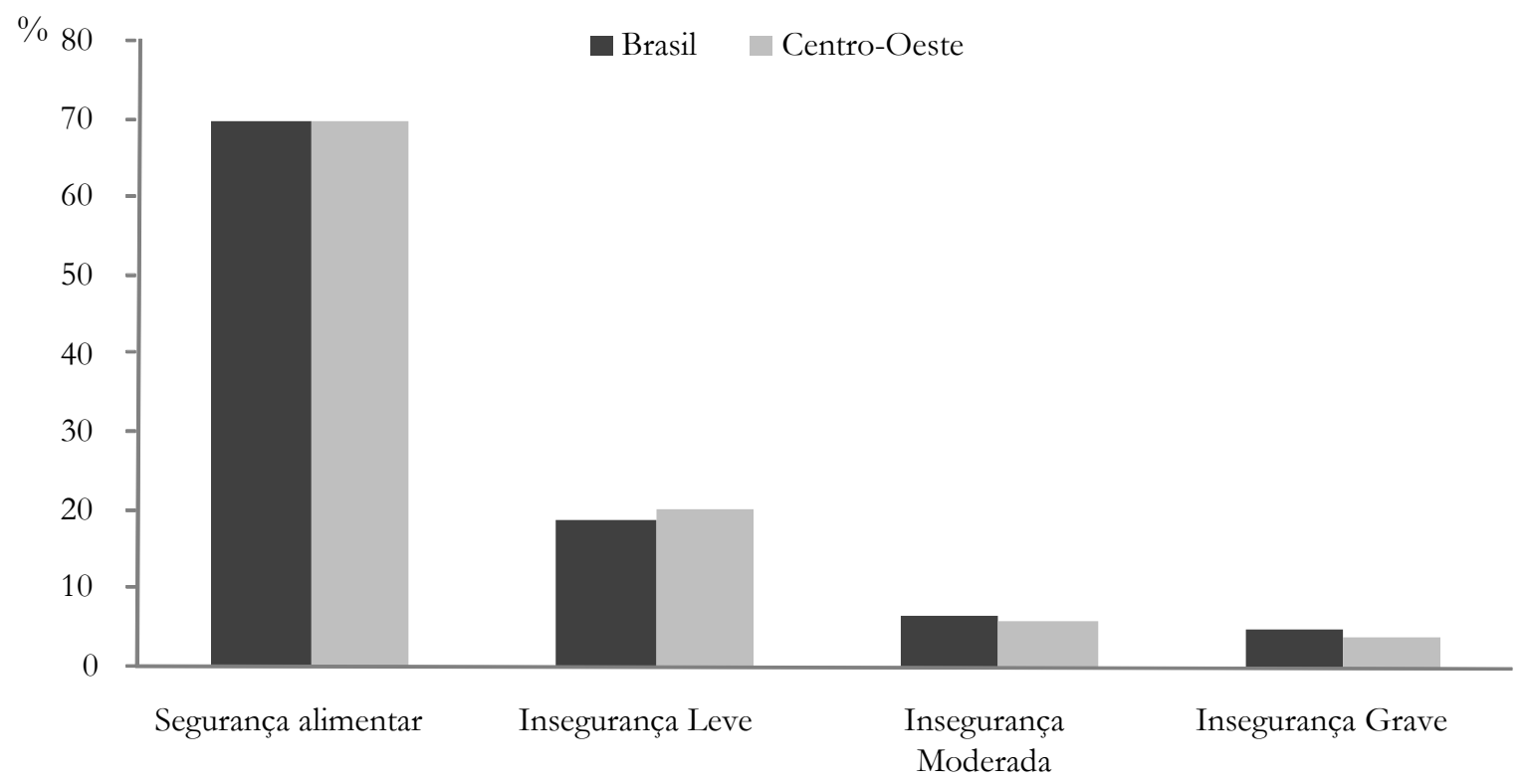

Figura 2. Prevalência da situação da segurança alimentar em domićlios particulares permanentes, na Região Centro-Oeste e no Brasil, 2009. Fonte: Instituto Brasileiro de Geografia e Estatística (IBGE), Pesquisa Nacional por Amostras de Domicílios (PNAD, 2009).

Tabela 2. Indicadores antropométricos em diferentes ciclos de vida. Região Centro-Oeste e Brasil, 2006-2009.

\begin{tabular}{|c|c|c|c|c|c|c|}
\hline \multicolumn{7}{|c|}{ Indicadores antropométricos para crianças entre 0 e 5 anos de idade ${ }^{a}$} \\
\hline & \multicolumn{2}{|c|}{ Déficit Altura para Idade ${ }^{\mathrm{b}}(\%)$} & \multicolumn{2}{|c|}{ Déficit Peso para Idadec $(\%)$} & \multicolumn{2}{|c|}{ Sobrepeso $^{\mathrm{d}}(\%)$} \\
\hline & \multicolumn{2}{|c|}{ Ambos os sexos } & \multicolumn{2}{|c|}{ Ambos os sexos } & \multicolumn{2}{|c|}{ Ambos os sexos } \\
\hline Centro-Oeste & \multicolumn{2}{|c|}{5,6} & \multicolumn{2}{|c|}{1,6} & \multicolumn{2}{|c|}{7,0} \\
\hline Brasil & \multicolumn{2}{|c|}{7,0} & \multicolumn{2}{|c|}{1,7} & \multicolumn{2}{|c|}{6,6} \\
\hline \multicolumn{7}{|c|}{ Indicadores antropométricos para adolescentes entre 10 e 19 anos de idade } \\
\hline & \multicolumn{2}{|c|}{ Déficit de Peso $^{f}(\%)$} & \multicolumn{2}{|c|}{ Excesso de Pesog $(\%)$} & \multicolumn{2}{|c|}{ Obesidadeh $(\%)^{2}$} \\
\hline & Masculino & Feminino & Masculino & Feminino & Masculino & Feminino \\
\hline Centro-Oeste & 3,3 & 2,8 & 23,9 & 20,0 & 5,5 & 3,7 \\
\hline Brasil & 3,7 & 3,0 & 21,5 & 19,4 & 5,8 & 4,0 \\
\hline \multicolumn{7}{|c|}{ Indicadores antropométricos para população com 20 ou mais anos de idade } \\
\hline & \multicolumn{2}{|c|}{ Déficit de Peso $^{\mathrm{i}}(\%)$} & \multicolumn{2}{|c|}{ Excesso de Pesoi $(\%)$} & \multicolumn{2}{|c|}{ Obesidadek $(\%)^{\circ}$} \\
\hline & Masculino & Feminino & Masculino & Feminino & Masculino & Feminino \\
\hline Centro-Oeste & 2,0 & 4,0 & 51,0 & 45,6 & 13,3 & 16,3 \\
\hline Brasil & 1,8 & 3,6 & 50,1 & 48,0 & 12,5 & 16,9 \\
\hline
\end{tabular}

a Pesquisa Nacional sobre Demografia e Saúde (PNDS), 2006/2007; b Altura/Idade $<-2$ escores $Z$; c Peso/Idade $<-2$

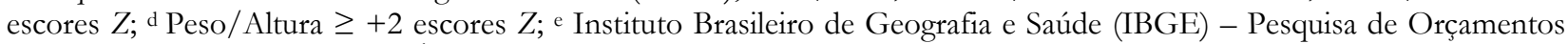
Familiares (POF), 2008-2009; ${ }^{\mathrm{f}}$ Índice de Massa Corporal (IMC) para idade e sexo $>+1$ escores $Z ;{ }^{\mathrm{g}}$ IMC para idade e sexo $>+2$ escores $Z$; ${ }^{\text {h }}$ IMC para idade $<-2$ escores $Z ;{ }^{\mathrm{i}} \mathrm{IMC}<18,5 \mathrm{~kg} / \mathrm{m}^{2 ;}$; IMC $\geq 25,0 \mathrm{~kg} / \mathrm{m}^{2} ; \mathrm{k}$ IMC $\geq 30,0 \mathrm{~kg} / \mathrm{m}^{2}$. 
$\mathrm{CO}$ apresentou a segunda menor prevalência de déficit de $\mathrm{A} / \mathrm{I}$ e $\mathrm{P} / \mathrm{I}$, se comparado às outras regiões, ficando atrás apenas do Sudeste para os dois indicadores. De todo modo, destaca-se que as prevalências encontradas de déficit de peso e altura configuram baixa exposição à desnutrição, por se apresentarem abaixo de 10\% [23]. Por outro lado, ainda de acordo com os dados da PNDS [18], verifica-se que a frequência de crianças com excesso de peso no CO $(7 \%)$ é maior do que a média nacional $(6,6 \%)$. A região fica atrás apenas do Sul, que apresenta prevalência de sobrepeso acima de $8 \%$ (dados não demonstrados).

Em relação à avaliação do IMC para idade de adolescentes, o CO apresentou menor prevalência de déficit de peso se comparado à prevalência nacional, em ambos os sexos [19]. A prevalência de excesso de peso para ambos os sexos na Região CO foi superior à nacional, representando a terceira região com maior prevalência no país, sendo inferior apenas ao Sul e Sudeste. Ademais, o CO apresentou a terceira maior prevalência de obesidade do país, estando atrás das Regiões Sul e Sudeste, para ambos os sexos [19]. Com base nesses dados, observa-se que o estado nutricional de adolescentes na Região $\mathrm{CO}$ não apresenta prevalências extremas de desnutrição e sobrepeso quando comparado às demais regiões (Tabela 2).

Os adultos de ambos os sexos da Região CO, segundo a POF (2008-2009), apresentaram uma maior prevalência de déficit de peso classificada pelo IMC, comparando com o Brasil (Tabela 2); sendo a segunda região com maior prevalência (atrás apenas da Região Nordeste).

Ressalta-se que prevalências de déficits de peso inferiores a 5\% não devem ser tomadas como evidência de exposição de populações adultas à desnutrição, uma vez que em populações não expostas a deficiências nutricionais é usual encontrar uma frequência de 3\% a $5 \%$ de adultos com IMC inferior a $18,5 \mathrm{~kg} / \mathrm{m}^{2}$, os quais correspondem à fração de indivíduos constitucionalmente magros, existentes em qualquer população [24]. Com isso, verifica-se a não exposição da população brasileira e da Região CO à desnutrição.

Em relação ao excesso de peso e obesidade, a população masculina da Região CO apresentou maior prevalência que o país como um todo e o sexo feminino, menor prevalência (Tabela 2). A Região CO apresentou a menor prevalência de excesso de peso entre as mulheres e a segunda maior prevalência de obesidade entre os homens (atrás apenas da Região Sul), se comparada às demais regiões ${ }^{[19]}$.

Dados apresentados no relatório do sistema Vigilância de Fatores de Risco e Proteção para Doenças Crônicas por Inquérito Telefônico (VIGITEL, 2008) indicam que as maiores frequências de excesso de peso foram observadas, no caso de homens, em Rio Branco $(56,3 \%)$, Campo Grande $(54,6 \%)$ e Porto Alegre $(54,1 \%)$ e, no caso de mulheres, em Cuiabá (44,8\%), Curitiba e Porto Alegre (44,7\%). Dessa forma, observase que as capitais do Mato Grosso do Sul e do Mato Grosso aparecem entre as cidades com maiores percentuais de excesso de peso [25].

Finalmente, há que se notar que a avaliação do estado nutricional da população por meio da antropometria em todas as etapas do curso da vida focaliza, primordialmente, alterações do balanço energético, não permitindo o diagnóstico de todos os distúrbios da nutrição. Dessa forma, indivíduos eutróficos podem apresentar distúrbios nutricionais não mensuráveis por meio da antropometria, o que pode aumentar o número de pessoas em situação de insegurança nutricional.

\section{Baixo peso ao nascer}

De acordo com dados do Sistema de Informações de Nascidos Vivos (SINASC) 2006 [20], a Região CO apresenta uma prevalência de $7,7 \%$ das crianças nascidas vivas com peso menor que $2.500 \mathrm{~g}$ (baixo peso ao nascer); prevalência menor que a encontrada no Brasil, de $8,2 \%$, sendo a terceira região com a maior proporção de recém-nascidos de baixo peso ao nascer (atrás das Regiões Sudeste e Sul). Ressalta-se que as maiores prevalências de baixo peso ao nascer encontradas nas Regiões Sul e Sudeste não condizem com os demais indicadores de estado nutricional e acesso ao pré-natal. É possível que essa diferença seja decorrente de subnotificação nas demais regiões ou de erro na alimentação dos dados nas regiões menos desenvolvidas, nas quais os recémnascidos com baixo peso que vão a óbito após o parto podem ter sido classificados como natimortos [26,27].

Dados anteriores do Brasil e de outros países também demonstraram menores percentuais de baixo peso ao nascer em áreas geográficas de menor desenvolvimento socioeconômico [27]. Esse paradoxo foi verificado na comparação de duas coortes de nascimentos: 2.839 recém-nascidos em Ribeirão Preto (São Paulo) em 1994 e 2.439 em São Luís (Maranhão) 
em 1997/98, onde se verificou que o tabagismo materno foi o fator de risco mais importante e capaz de explicar a diferença no baixo peso ao nascer entre as duas cidades [26]. O tabagismo seria um comportamento mais ligado a mulheres de áreas urbanas [27]. Além desse, existem outros fatores de risco para o baixo peso ao nascer, como o peso pré-gestacional inadequado, o ganho de peso insuficiente durante a gestação, a baixa estatura materna, a idade materna abaixo dos 18 anos ou acima dos 35 anos, intervalo interpartal e a atenção pré-natal [26,28].

\section{Prevalência do aleitamento materno}

De acordo com os dados da Pesquisa de Prevalência de Aleitamento Materno em Municípios Brasileiros realizada pelo Ministério da Saúde (MS), em 2010, a prevalência de crianças menores de 6 meses em aleitamento materno exclusivo nos municípios estudados na Região CO variou de 12,9\% (Senador Canedo, Goiás) a 50\% (Brasília/Distrito Federal, DF). De acordo com esta pesquisa, a maioria dos municípios na Região CO apresentou prevalências deste indicador inferiores à média nacional $(41,0 \%)^{[21]}$.

Em estudo pontual realizado no ano de 2004 em Cuiabá, durante um dia de campanha de vacinação [29], verificou-se prevalência de aleitamento materno exclusivo em crianças menores de 6 meses de 28,5\%, sendo que a oferta de líquidos foi o principal responsável pelo desmame precoce ${ }^{[30]}$.

Segundo dados da PNDS 2006, o percentual encontrado no CO de crianças menores de 60 meses que foram alguma vez amamentadas foi $97,7 \%$, maior do que o percentual nacional $(96,4 \%)$, sendo a segunda região com o maior percentual de crianças alguma vez amamentadas, perdendo somente para o Norte (98,2\%). Com relação à duração média do aleitamento materno, a PNDS 2006 mostra que a região em estudo apresenta 2,46 meses de aleitamento materno exclusivo, valor maior do que a média nacional (2,17 meses), e 6,9 meses de aleitamento materno total, duração menor do que a média nacional (7,59 meses) ${ }^{[18]}$.

\section{Acesso ao pré-natal}

O número de consultas de pré-natal recomendado pela OMS e pelo MS é de, no mínimo, 6 consultas ${ }^{[31}$. Ao avaliar o acesso ao pré-natal por meio do número de consultas realizadas em relação ao total de nascidos vivos, verifica-se que o acesso a 7 ou mais consultas de pré-natal foi maior na Região CO $(59,8 \%)$ do que no Brasil (53,6\%). Quando comparado às demais regiões, o CO fica em uma classificação intermediária, tendo o Sul e Sudeste uma maior cobertura e o Norte e Nordeste uma pior cobertura, segundo dados de 2005 [20].

O baixo acesso ao pré-natal pode contribuir para uma maior prevalência de baixo peso ao nascer, mortalidade infantil e redução do tempo de aleitamento materno. Todos esses fatores podem contribuir para agravar a insegurança alimentar e nutricional na Região CO.

\section{Taxa de mortalidade infantil}

No que diz respeito à mortalidade infantil, a taxa da Região CO é menor do que a do Brasil, perfazendo, respectivamente, 15,5 e 16,4 casos de óbitos de crianças nascidas vivas no primeiro ano de vida para cada 1000 nascidos vivos, segundo dados de 2006 [20]. De forma similar, em uma coorte constituída de 20.981 nascidos vivos em Goiânia (GO), a probabilidade de morte antes de completar um ano de vida foi de 16,3 óbitos por mil nascidos vivos. Nesse mesmo estudo, tiveram maior chance de mortalidade no período neonatal os nascidos em hospital públicoestatal, os prematuros e os com baixo peso ao nascer e, menor chance, os nascidos por cesariana. Além disso, os subgrupos expostos a um maior risco de mortalidade pós-neonatal foram: nascidos vivos de mãe sem instrução, os nascidos com baixo peso ao nascer ou em hospital público-estatal [32].

\section{Alimento seguro}

Os níveis de resíduos de agrotóxicos nos alimentos in natura que chegam à mesa do consumidor foram obtidos do Programa de Análise de Resíduos de Agrotóxicos (PARA, ANVISA - dados de serviço). Em 2008, foram monitoradas 17 culturas e no CO, participaram os Estados de Mato Grosso do Sul e de Goiás e o DF (Tabela 3).

Nota-se na Tabela 3 que os percentuais de amostras inadequadas de abacaxi, arroz, cebola, cenoura, feijão, laranja, maçã, pimentão e uva no CO foram maiores que os percentuais observados no país de modo geral, variando de $4 \%$ para o feijão até $58 \%$ para a cenoura. Vale ressaltar que tais culturas são de consumo frequente pela população brasileira. Por outro lado, não foram encontradas amostras de banana, 
Tabela 3. Proporção de amostras de alimentos contaminados por agrotóxicos na Região Centro-Oeste e no Brasil, segundo a cultura, 2008.

\begin{tabular}{lccccc}
\hline Alimentos & $\begin{array}{c}\text { Brasil } \\
(\mathbf{\%})\end{array}$ & $\begin{array}{c}\text { Centro-Oeste } \\
\mathbf{( \% )}\end{array}$ & $\begin{array}{c}\text { Mato Grosso do Sul } \\
\mathbf{( \% )}\end{array}$ & $\begin{array}{c}\text { Goiás } \\
\mathbf{( \% )}\end{array}$ & $\begin{array}{c}\text { Distrito Federal } \\
\mathbf{( \% )}\end{array}$ \\
\hline Abacaxi & 10 & 16 & 33 & 0 & 17 \\
Alface & 20 & 10 & 14 & 0 & 17 \\
Arroz & 4 & 7 & 11 & 11 & 0 \\
Banana & 1 & 0 & 0 & 0 & 0 \\
Batata & 2 & 0 & 0 & 0 & 0 \\
Cebola & 3 & 10 & 14 & 0 & 17 \\
Cenoura & 30 & 58 & 57 & 71 & 40 \\
Feijão & 3 & 4 & 0 & 0 & 10 \\
Laranja & 15 & 20 & 17 & 29 & 14 \\
Maçã & 4 & 5 & 14 & 0 & 0 \\
Mamão & 17 & 14 & 0 & 43 & 0 \\
Manga & 1 & 0 & 0 & 0 & 0 \\
Morango & 36 & 22 & 20 & 29 & 17 \\
Pimentão & 64 & 68 & 67 & 86 & 50 \\
Repolho & 9 & 5 & 14 & 0 & 0 \\
Tomate & 18 & 15 & 17 & 29 & 0 \\
Uva & 32 & 35 & 33 & 43 & 29 \\
\hline
\end{tabular}

Fonte: Programa de Análise de Resíduos de Agrotóxicos em Alimentos (PARA), da Agência Nacional de Vigilância Sanitária (ANVISA), 2008 (dados de serviço).

batata e manga inadequadas em relação à contaminação por agrotóxicos no CO.

Analisando-se os dados por Unidade Federativa, são verificados dados alarmantes de contaminação, a exemplo de $71 \%$ das amostras de cenoura e $86 \%$ das amostras de pimentão, encontrados em Goiás, por exemplo. A contaminação de alimentos por agrotóxicos contribui para a IA na região em estudo, podendo ocasionar agravos à saúde da população.

\section{Saneamento básico}

Segundo dados da PNAD 2005 [22], tanto o percentual de domicilios com rede geral de abastecimento de água quanto o percentual de domicílios com esgotamento sanitário no $\mathrm{CO}$ são inferiores aos percentuais nacionais (Tabela 4). A exceção foi o DF, que também possui o maior percentual de domicílios com serviço de coleta de lixo, direto ou indireto. Nesse sentido, destaca-se que a cobertura de coleta de lixo nos domicílios do CO encontra-se em melhor situação do que a cobertura nacional, com exceção do Mato Grosso.

\section{Dimensão 6: Educação}

Em pessoas com 15 anos de idade ou mais, a taxa de analfabetismo e analfabetismo funcional (menos de quatro anos de estudo), na população brasileira e no CO, pode ser observada na Tabela 5 . Nota-se que as taxas de analfabetismo e de analfabetismo funcional da população do $\mathrm{CO}$ foram menores do que as da população do país de modo geral, com destaque para o DF cujos valores das taxas de analfabetismo e analfabetismo funcional foram aproximadamente metade dos valores observados nos outros Estados da região [22].

Quando se avalia o percentual de crianças e adolescentes entre 5 e 17 anos que não frequentava a escola, a situação do CO (9,4\%) encontra-se pior que a do Brasil (8,8\%), com exceção do DF, onde se verifica que apenas 6,5\% das crianças e adolescentes entre 5 e 17 anos não frequentavam a escola [22].

Segundo a PNAD 2005 [22], a média de anos de estudo das pessoas com 10 anos ou mais de idade do CO (6,9 anos) é muito próxima da média observada no 
Tabela 4. Percentual de domicílios atendidos por saneamento básico no total de domicílios particulares permanentes, na Região Centro-Oeste e no Brasil, 2005.

\begin{tabular}{|c|c|c|c|}
\hline Regiões & $\begin{array}{c}\text { Domicílios com rede geral } \\
\text { de abastecimento de água } \\
(\%)\end{array}$ & $\begin{array}{c}\text { Domicílios com } \\
\text { esgotamento sanitário } \\
(\%)\end{array}$ & $\begin{array}{c}\text { Domicílios com serviço } \\
\text { de coleta de lixo } \\
(\%)\end{array}$ \\
\hline Brasil & 92,5 & 66,2 & 97,1 \\
\hline Centro-Oeste & 88,4 & 40,0 & 98,1 \\
\hline Mato Grosso do Sul & 92,8 & 13,7 & 98,5 \\
\hline Mato Grosso & 85,9 & 22,0 & 94,0 \\
\hline Goiás & 85,4 & 37,2 & 99,0 \\
\hline Distrito Federal & 94,0 & 87,4 & 99,6 \\
\hline
\end{tabular}

Fonte: Instituto Brasileiro de Geografia e Estatística (IBGE), Pesquisa Nacional por Amostras de Domicílios (PNAD), 2005).

Tabela 5. Percentual de analfabetismo e analfabetismo funcional nas pessoas com 15 anos ou mais de idade, por sexo e situação do domicílio, na Região Centro-Oeste e no Brasil, 2005.

\begin{tabular}{|c|c|c|c|c|c|}
\hline \multirow{2}{*}{$\begin{array}{c}\text { Proporção de analfabetismo no } \\
\text { Brasil e no Centro-Oeste }(\%)\end{array}$} & \multirow{2}{*}{$\begin{array}{l}\text { Total } \\
(\%)\end{array}$} & \multicolumn{2}{|c|}{ Sexo } & \multicolumn{2}{|c|}{ Situação do domicílio } \\
\hline & & $\begin{array}{c}\text { Homens } \\
(\%)\end{array}$ & $\begin{array}{c}\text { Mulheres } \\
(\%)\end{array}$ & $\begin{array}{c}\text { Urbana } \\
(\%)\end{array}$ & $\begin{array}{c}\text { Rural } \\
(\%)\end{array}$ \\
\hline Brasil & 11,0 & 11,3 & 10,8 & 8,4 & 25,0 \\
\hline Centro-Oeste & 8,9 & 8,7 & 9,1 & 7,9 & 15,4 \\
\hline Mato Grosso do Sul & 9,1 & 7,9 & 10,2 & 8,6 & 11,8 \\
\hline Mato Grosso & 9,7 & 9,7 & 9,8 & 8,2 & 14,9 \\
\hline Goiás & 10,2 & 10,2 & 10,2 & 9,1 & 18,5 \\
\hline Distrito Federal & 4,7 & 4,6 & 4,7 & 4,4 & 10,1 \\
\hline $\begin{array}{c}\text { Proporção de analfabetismo } \\
\text { funcional no Brasil e no } \\
\text { Centro-Oeste }(\%)\end{array}$ & $\begin{array}{c}\text { Total } \\
(\%)\end{array}$ & $\begin{array}{l}\text { Homens } \\
\quad(\%)\end{array}$ & $\begin{array}{l}\text { Mulheres } \\
(\%)\end{array}$ & $\begin{array}{c}\text { Urbana } \\
(\%)\end{array}$ & $\begin{array}{c}\text { Rural } \\
(\%)\end{array}$ \\
\hline Brasil & 23,5 & 24,1 & 23,0 & 19,3 & 45,8 \\
\hline Centro-Oeste & 21,4 & 21,9 & 20,9 & 18,9 & 36,8 \\
\hline Mato Grosso do Sul & 23,2 & 22,0 & 24,3 & 21,4 & 33,6 \\
\hline Mato Grosso & 23,9 & 25,0 & 22,8 & 19,6 & 38,6 \\
\hline Goiás & 23,6 & 24,4 & 22,9 & 21,4 & 39,5 \\
\hline Distrito Federal & 11,1 & 11,3 & 10,9 & 10,7 & 18,4 \\
\hline
\end{tabular}

Fonte: Instituto Brasileiro de Geografia e Estatística (IBGE), Pesquisa Nacional por Amostras de Domicílios (PNAD), 2005. 
país (6,6 anos). Entretanto, a média verificada no DF (8,5 anos) é maior do que as observadas nos outros Estados da Região CO, as quais se situam em torno de 6,6 anos.

Assim, de acordo com os indicadores da dimensão de educação da SAN, o DF apresenta população em situação educacional privilegiada em comparação com os outros Estados do CO, melhorando, dessa forma, os indicadores da região.

\section{Dimensão 7: Políticas públicas que promovem a Segurança Alimentar e Nutricional}

Segundo dados do Ministério de Desenvolvimento Social e Combate à Fome (MDS) [33], diversas políticas públicas de SAN incidiam sobre a população da Região Centro-Oeste. As principais são apresentadas na Tabela 6 , indicando o alcance populacional em novembro de 2009 por Unidade da Federação e região como um todo. Sem dúvida o Programa Bolsa Familia (PBF) é o de cobertura mais expressiva no Centro-Oeste e no Brasil, garantindo uma renda mínima todos os meses para 686 mil famílias. A estrutura normativa do programa (Decreto no 5.209, de 17 de setembro de 2004) descreve como um dos objetivos do PBF "combater a fome e promover a Segurança Alimentar e Nutricional".

Dentre os outros programas investigados apenas o de Cestas de Alimentos e o Programa de Aquisição de Alimentos da agricultura familiar (PAA) atingem mais de 100 mil pessoas (cada) na região. Existe uma estimativa de 789 mil Familias Pobres no Centro-Oeste (Perfil Bolsa Família segundo PNAD 2006, extraída da Matriz de Informações Sociais do MDS). Assim sendo, se os diferentes programas não forem cumulativos, mas sim alcançarem públicos diferentes, o contingente populacional coberto pelos três primeiros programas chega próximo à estimativa global de famílias pobres. Supondo-se 3,5 pessoas por familia haveria um adicional de 82 mil famílias sendo atendidas pelos alimentos das Cestas e do PAA, além das 686 mil do PBF, o que indica uma situação razoável em termos de cobertura de políticas sociais promotoras de SAN e do DHAA.

\section{CONCLUSÕES}

Pode-se concluir que, apesar do CO ocupar posição de destaque na produção agropecuária e apresentar bom desempenho em alguns indicadores de
SAN quando comparado aos valores nacionais (menor prevalência de baixo peso ao nascer, maior acesso ao pré-natal, menor taxa de mortalidade infantil, menor taxa de analfabetismo em adultos), é uma região de contrastes marcantes e muitos fatores apontam para situações que podem acarretar insegurança alimentar e nutricional em parcelas da população da região.

Nesse sentido, destaca-se que a concentração da produção, a ocorrência de grandes propriedades, a desigualdade na distribuição de renda, a baixa disponibilidade de calorias e carne bovina, o alto gasto com a alimentação e o comprometimento de alguns indicadores de saneamento básico e de educação são fatores que podem contribuir para um quadro de insegurança alimentar e nutricional. Por outro lado, indicadores como a alta proporção de gastos com alimentação fora do domićlio, o baixo consumo de frutas e hortaliças, o alto consumo de óleos e gorduras e a contaminação por agrotóxico em alguns alimentos de largo consumo pela população, entre outros fatores desfavoráveis encontrados na região em estudo, podem contribuir para escolhas alimentares inadequadas e para o aumento da prevalência de doenças crônicas nãotransmissíveis.

Este estudo apontou a utilidade da Matriz de indicadores de SAN, proposta pelo CONSEA, no monitoramento da realização progressiva do DHAA e da situação de SAN da população brasileira e de regiões específicas do país, por ser abrangente e utilizar dados disponíveis em órgãos do governo ou gerados por meio de pesquisas nacionais. Estudos longitudinais são necessários para monitorar a evolução dos indicadores que constam na Matriz de monitoramento da SAN e seus determinantes na Região CO ao longo do tempo.

Por oportuno, cabe destacar que o enfoque ampliado de SAN demanda mudanças importantes para o seu enfrentamento. Assim, acredita-se que a melhoria do quadro de IA na Região Centro-Oeste, bem como no Brasil, somente será possível com a interação e colaboração entre os diversos órgãos e entidades envolvidos com a agropecuária, a proteção social, a educação, a saúde, o saneamento básico, dentre outros. Nesta perspectiva, aponta-se para a necessidade do fortalecimento de dois pilares: o contínuo aperfeiçoamento de instrumentos de monitoramento da situação de SAN e a priorização de ações que visem à redução da desigualdade social, uma vez que, como observado, esta característica gera maior agravamento da IA na Região Centro-Oeste. 
Tabela 6. Políticas públicas que promovem a Segurança Alimentar e Nutricional na Região Centro-Oeste, 2009.

\begin{tabular}{|c|c|c|c|c|c|}
\hline $\begin{array}{l}\text { Políticas públicas de Segurança Alimentar e } \\
\text { Nutricional }\end{array}$ & $\begin{array}{l}\text { Centro- } \\
\text { Oeste }\end{array}$ & $\begin{array}{c}\text { Mato } \\
\text { Grosso } \\
\text { do Sul }\end{array}$ & $\begin{array}{l}\text { Mato } \\
\text { Grosso }\end{array}$ & Goiás & $\begin{array}{l}\text { Distrito } \\
\text { Federal }\end{array}$ \\
\hline \multicolumn{6}{|l|}{ Programa Bolsa Família (PBF) } \\
\hline № de famílias atendidas por mês & 686.400 & 129.200 & 168.800 & 308.900 & 79.500 \\
\hline Cobertura de familias pobres com perfil $\mathrm{PBF}^{a}$ & $(87,0 \%)$ & $(87,7 \%)$ & $(94,4 \%)$ & $(87,0 \%)$ & $(73,5 \%)$ \\
\hline
\end{tabular}

\section{Cestas de Alimentos}

№ de Cestas por mês

$\begin{array}{lllll}134.900 & 69.500 & 41.601 & 22.900 & 900 \\ 177.00 & 102.000 & 39.200 & 33.900 & 1.900\end{array}$

\section{Programa de Aquisição de Alimentos (PAA)}

№ de agricultores por mês

$\begin{array}{rrrrr}1.418 & 534 & 359 & 337 & 188 \\ 112.100 & 30.200 & 49.000 & 27.300 & 5.600\end{array}$

População atendida pelos alimentos PAA por mês

\section{Restaurante Popular ou Cozinha comunitária}

№ de equipamentos

15

$1^{\mathrm{b}}$

6

7

1

População atendida nos equipamentos por dia

6.635

2.035

1.600

3.000

\section{Banco de Alimentos}

№ de equipamentos

5

3

1

1

0

\section{Carteira Indígena}

№ de projetos de desenvolvimento sustentado

$56 \quad 31 \quad 25$

0

0

a Calculada a partir da estimativa de Famílias Pobres - Perfil Bolsa Família (PNAD, 2006), extraída da Matriz de Informações Sociais do Ministério de Desenvolvimento Social e Combate à Fome (MDS); b Unidade em construção. Fonte: Ministério do Desenvolvimento Social e Combate à Fome ${ }^{[33]}$. 


\section{REFERÊNCIAS}

[1] Santos SMC, Santos LMP. Avaliação de políticas públicas de segurança alimentar e combate à fome no período de 1995-2002. 1 - Abordagem metodológica. Cad Saúde Pública. 2007;23(5):1029-40.

[2] Maluf RS, Menezes F, Valente FL. Contribuição ao Tema da Segurança Alimentar no Brasil. Cadernos de Debate. 1996;4:66-88.

[3] Belik W. Perspectivas para segurança alimentar e nutricional no Brasil. Saude Soc. 2003;12(1):12-20.

[4] Food and Agriculture Organization. Rome Declaration on World Food Security. Roma, 1996 [acesso em 07 jul 2009]. Disponível em: http://www.fao.org/docrep/003/w3613e/w3613e00.HTM

[5] Conselho de Segurança Alimentar e Nutricional. Relatório Final da II Conferência Nacional de Segurança Alimentar e Nutricional. 2004 [acesso em 07 jul 2009]. Disponível em: https://www.planalto.gov.br/Consea/static/documentos/O utros/IIConferencia.pdf

[6] Conselho de Segurança Alimentar e Nutricional. Relatório Final da III Conferência Nacional de Segurança Alimentar e Nutricional. 2007 [acesso em 07 jul 2009]. Disponível em: https://www.planalto.gov.br/consea/3conferencia/static/in dex.htm

[7] Burlandy L. Transferência condicionada de renda e Segurança Alimentar e Nutricional. Ciência Saúde Colet. 2007;12(6):1441-51.

[8] Batista Filho M. O Brasil e a segurança alimentar. Rev Bras Saúde Matern Infant. 2007;7(2):121-22.

[9] Leão MM. Fome e insegurança alimentar no Distrito Federal [dissertação]. Brasília: Universidade de Brasília; 2005.

[10] Instituto Brasileiro de Geografia e Estatística. Pesquisa Nacional por Amostra de Domicílios - PNAD. Segurança Alimentar 2004/2009. Rio de Janeiro: IBGE; 2010.

[11] Enes CC, Silva MV. A Alimentação das famílias do Centro-Oeste brasileiro: subsídios para ações de Segurança Alimentar e Nutricional. Seg Alim Nutr. 2008;15(2):46-57.

[12] Conselho de Segurança Alimentar e Nutricional. Construindo um sistema de monitoramento da realização progressiva do Direito Humano à Alimentação Adequada (DHAA), no contexto do Sistema Nacional de Segurança Alimentar e Nutricional (SISAN). Brasília: CONSEA; 2007 [acesso em 07 jul 2009]. Disponível em: https://www.planalto.gov.br/consea
[13] Companhia Nacional de Abastecimento. Safra de Grãos; 2009 [acesso em 27 jun 2009]. Disponível em: http://www.conab.gov.br

[14] Instituto Brasileiro de Geografia e Estatística. Estatística da produção pecuária. Rio de Janeiro: IBGE; 2009.

[15] Instituto Brasileiro de Geografia e Estatística. Pesquisa de Orçamentos Familiares - POF, 2008/2009: Despesas, Rendimentos e Condições de Vida. Rio de Janeiro: IBGE; 2010.

[16] Instituto Brasileiro de Geografia e Estatística. Pesquisa Nacional por Amostra de Domicílios - PNAD, 2007. Rio de Janeiro: IBGE; 2008.

[17] Instituto Brasileiro de Geografia e Estatística. Pesquisa de Orçamentos Familiares - POF, 2002/2003. Análise da disponibilidade domiciliar de alimentos e do estado nutricional no Brasil. Rio de Janeiro: IBGE; 2004.

[18] Ministério da Saúde. Pesquisa Nacional de Demografia e Saúde da criança e da mulher - PNDS, 2006. Relatório Final, Brasília; 2008.

[19] Instituto Brasileiro de Geografia e Estatística. Pesquisa de Orçamentos Familiares no Brasil - POF, 2008/2009. Antropometria e estado nutricional de crianças, adolescentes e adultos. Rio de Janeiro: IBGE; 2010.

[20] Ministério da Saúde. Sistema de Vigilância à Saúde SVS. Sistema de Informações de Nascidos Vivos (SINASC); Indicadores e dados básicos (IDB) / DATASUS [acesso em 27 jun 2009]. Disponível em: http://www.datasus.gov.br

[21] Ministério da Saúde. Secretaria de Atenção à Saúde. Departamento de Ações Programáticas e Estratégicas Pesquisa de Prevalência de Aleitamento Materno em Municípios Brasileiros. Situação do Aleitamento Materno em 227 municípios brasileiros. Brasília - DF: MS; 2010.

[22] Instituto Brasileiro de Geografia e Estatística. Pesquisa Nacional por Amostra de Domicílios - PNAD, 2005. Síntese de Indicadores Sociais. Rio de Janeiro: IBGE; 2006.

[23] De Onis M, Monteiro CA, Akré J, Clugston G. The worldwide magnitude of protein-energy malnutrition: an overview from the WHO Global Database on Child Growth. Bull World Health Org. 1993;71(6):703-12.

[24] World Health Organization. Physical status: the use and interpretation of anthropometry: report of a WHO Expert Committee. Geneva, 1995. 462 p. (WHO Technical Report Series, 854) [acesso em 07 jul 2010]. Disponível em: http:// www.who.int/childgrowth/publications/physical status/en/ index.html 
[25] Ministério da Saúde. Sistema de Vigilância à Saúde SVS. Secretaria de Gestão Estratégica e Participativa. Vigitel Brasil 2008: Vigilância de Fatores de Risco e Proteção para Doenças Crônicas por Inquérito Telefônico. Brasillia: MS; 2009.

[26] Silva MAS, Bettiol H, Barbieri MA, Brito LGO, Pereira MM, Aragão VMF, Ribeiro VS. Which factors could explainthe low birth weight paradox? Rev Saúde Públ. 2006;40(4):648-55.

[27] Andrade CLT, Szwarcwald CL, Castilho EA. Baixo peso ao nascer no Brasil de acordo com as informações sobre nascidos vivos do Ministério da Saúde, 2005. Cad Saúde Pública. 2008;24(11):2564-72.

[28] Franceschini SCC, Priore SE, Pequeno NPF, Silva DG, Sigulem DM. Fatores de risco para o baixo peso ao nascer em gestantes de baixa renda. Rev Nutr. 2003;16(2):171-79.

[29] Silva SM, Brunken GS, França GVA, Escuder MM, Venancio SI. Evolução do aleitamento materno em uma
Capital da Região Centro-Oeste do Brasil entre 1999 e 2004. Cad Saúde Pública. 2007;23(7):1539-46.

[30] Brunken GS, Silva SM, França GVA, Escuder MM, Venancio SI. Risk factors for early interruption of exclusive breastfeeding and late introduction of complementary foods among infants in midwestern Brazil. J Pediatr. 2006; 82(6):445-51.

[31] Alencar Jr CA. Projeto Diretrizes. Assistência Pré-Natal. Associação Médica Brasileira e Conselho Federal de Medicina; 2001.

[32] Morais Neto OL, Barros MBA. Fatores de risco para mortalidade neonatal e pós-neonatal na Região Centro-Oeste do Brasil: linkage entre bancos de dados de nascidos vivos e óbitos infantis. Cad Saúde Pública. 2000;16(2):477-85.

[33] Ministério do Desenvolvimento Social e Combate à Fome. MDS em números [acesso em 21 fev 2010]. Disponível em: http://www.mds.gov.br/sites/mds-emnumeros/paginas/estados 